\title{
Comparing Immune-Tumor Growth Models with Drug Therapy using Optimal Control
}

\author{
Marisa C. Martins*, Ana Maria A.C. Rocha*, M. Fernanda P. Costa ${ }^{\dagger}$ and Edite \\ M.G.P. Fernandes* \\ ${ }^{*}$ Algoritmi Research Centre, University of Minho, 4710-057 Braga, Portugal \\ ${ }^{\dagger}$ Centre of Mathematics, University of Minho, 4710-057 Braga, Portugal
}

\begin{abstract}
In this paper we compare the dynamics of three tumor growth models that include an immune system and a drug administration therapy using optimal control. The objective is to minimize a combined function of the total of tumor cells over time and a chemotherapeutic drug administration.
\end{abstract}

Keywords: Tumor Growth Model, Immune system, Optimal control

PACS: $02.60 . P n$

\section{INTRODUCTION}

A great deal of resources have been devoted to study the dynamics and interactions of tumor and normal cells aiming to devise tumor control strategies. Cells that divide and grow uncontrollably form malignant tumors, like in cancer. Cancer treatments aim to remove as efficiently as possible the tumor without damaging other cells of the body. Besides surgery, there are other types of treatments: chemotherapy, radiotherapy and immunotherapy. The choice of the most adequate therapy depends on the location of the tumor and on the stage of the disease. Chemotherapy and radiotherapy have the most negative effect on normal cells.

Mathematics has been contributing over the years to the analysis and simulation of tumor growth models. Studies that model the dynamics of healthy and cancer cells under drug therapy are also available in the literature. Tumor, immune and normal cells are considered in the tumor growth model presented in $[1,2]$. The authors analyze competition between normal cells and tumor cells and consider the role of chemotherapeutic drug. A tumor growth model with the effect of tumor-immune interaction and chemotherapeutic drug is also presented and studied in [3]. In [4], the radiotherapy is analyzed as the primary cancer strategy. Tumor and normal cells are competing in the model presented in [5].

The herein analyzed models extend some of the ideas presented in Pinho et al. [5] and represent a simplified version of the model in De Pillis et al. [2], and at the same time deals with a chemotherapy treatment. The basic model considers tumor, normal and immune cells and is represented by the following set of ordinary differential equations:

$$
\begin{array}{ll}
\frac{d T(t)}{d t}= & r_{T} T(t)\left(1-b_{T} T(t)\right)-c_{T} I(t) T(t) \\
\frac{d N(t)}{d t}= & r_{N} N(t)\left(1-b_{N} N(t)\right)-c_{N} N(t) I(t) \\
\frac{d I(t)}{d t}= & c_{N} N(t) I(t)-b_{I} I(t)-c_{I} I(t) T(t)
\end{array}
$$

for $0 \leq t \leq t_{f}$, with initial conditions

$$
T(0)=T_{0} \geq 0, N(0)=N_{0} \geq 0, I(0)=I_{0} \geq 0,
$$

where the variables and parameters have the following meaning: 


$\begin{aligned} t- & \text { time instant (in days) } \\ T(t)- & \text { number of tumor cells at time } t \\ N(t)- & \text { number of normal cells at time } t \\ I(t)- & \text { number of immune cells at time } t \\ r_{T}- & \text { per capita growth rate of the tumor cells } \\ r_{N}- & \text { per capita growth rate of the normal cells }\end{aligned}$

$c_{I}-\quad$ rate of loss of immune cells due to the tumor cells

$c_{T}-$ rate of loss of tumor cells due to the immune cells

$c_{N}-$ rate of conversion of normal to immune cells

$b_{T}-$ reciprocal carrying capacity of the tumor cells

$b_{N}-$ reciprocal carrying capacity of the normal cells

$b_{I}-$ reciprocal carrying capacity of the immune cells

We note that both $T(t)$ and $N(t)$ in the model in (1) exhibit logistic growth law (in the absence of immune cells), $T(t)$ and $I(t)$ compete in a predator-prey fashion, and the immune cells will die at a per capita rate $b_{I}$, in the absence of any tumor.

\section{TUMOR MODELS}

We now add the effect of the chemotherapeutic drug on the system. We denote by $D(t)$ the amount of drug at the tumor site at time $t$ and we assume that the drug kills all type of cells, although at different rates. The control $u(t)$ represents the dose of drug given in the chemotherapy treatment and the functions that represent the effect of the drug administration on the cell populations are by now considered killing-type functions where the saturation is applied only on the agent, resulting in the model with four ordinary differential equations (with four state variables and one control variable):

$$
\begin{aligned}
& \frac{d T(t)}{d t}=r_{T} T(t)\left(1-b_{T} T(t)\right)-c_{T} I(t) T(t)-a_{T} \frac{D(t) T(t)}{d_{T}+D(t)} \\
& \frac{d N(t)}{d t}=r_{N} N(t)\left(1-b_{N} N(t)\right)-c_{N} N(t) I(t)-a_{N} \frac{D(t) N(t)}{d_{N}+D(t)} \\
& \frac{d I(t)}{d t}=c_{N} N(t) I(t)-b_{I} I(t)-c_{I} I(t) T(t)-a_{I} \frac{D(t) I(t)}{d_{I}+D(t)} \\
& \frac{d D(t)}{d t}=u(t)-d_{D} D(t)
\end{aligned}
$$

for $0 \leq t \leq t_{f}$, where $d_{D}$ gives the per capita decay rate of the drug (from the system) and $D(0)=D_{0} \geq 0$. The other parameters are as follows: $a_{T}$ is the killing rate of the drug for the tumor cells and $d_{T}$ is the tumor threshold rate, $a_{N}$ is the killing rate of the drug for the normal cells and $d_{N}$ the threshold rate, $a_{I}$ is the killing rate of the drug for the immune cells and $d_{I}$ the threshold rate.

We note that the first presented model (3) as well as the other two presented below have (i) an immune cell population that grows by the presence of the tumor or normal cells and destroys tumor cells; (ii) competition terms between normal and immune cells and between tumor and immune cells; (iii) an optimal drug therapy that minimizes a combination of the tumor cell population and the drug administration over time.

When it is assumed that the presence of tumor cells stimulates the immune response, a positive nonlinear growth term for the immune cells is considered as it is shown in the third equation of the following model:

$$
\begin{array}{ll}
\frac{d T(t)}{d t}= & r_{T} T(t)\left(1-b_{T} T(t)\right)-c_{T} I(t) T(t)-a_{T} D(t) T(t) \\
\frac{d N(t)}{d t}= & r_{N} N(t)\left(1-b_{N} N(t)\right)-c_{N} N(t) I(t)-a_{N} D(t) N(t) \\
\frac{d I(t)}{d t}= & \frac{\rho T(t)}{\alpha+T(t)} I(t)-b_{I} I(t)-c_{I} I(t) T(t)-a_{I} D(t) I(t) \\
\frac{d D(t)}{d t}=u(t)-d_{D} D(t)
\end{array}
$$

where $\rho, \alpha>0$. In this second model, the negative effect of the chemotherapeutic drug on tumor, normal and immune cells is represented by simpler relations as shown in the last terms of the three first equations of the system (4). 
The third model herein analyzed uses an exponential curve as the response to the drug cell killing, resulting in the following system:

$$
\begin{array}{ll}
\frac{d T(t)}{d t}= & r_{T} T(t)\left(1-b_{T} T(t)\right)-c_{T} I(t) T(t)-a_{T}(1-\exp (-D(t))) T(t) \\
\frac{d N(t)}{d t}= & r_{N} N(t)\left(1-b_{N} N(t)\right)-c_{N} N(t) I(t)-a_{N}(1-\exp (-D(t))) N(t) \\
\frac{d I(t)}{d t}= & \frac{\rho T(t)}{\alpha+T(t)} I(t)-b_{I} I(t)-c_{I} I(t) T(t)-a_{I}(1-\exp (-D(t))) I(t) \\
\frac{d D(t)}{d t}=u(t)-d_{D} D(t)
\end{array}
$$

\section{OPTIMAL CONTROL}

In this section, we consider the problem of finding the optimal control in the tumor-immune mathematical model. In this optimal control problem we seek an optimal control $u^{*}$ such that

$$
J\left(u^{*}\right)=\min \{J(u): u \in U\}
$$

where $U=\left\{u: u\right.$ is measurable $\left., 0 \leq u(t) \leq 1, t \in\left[0, t_{f}\right]\right\}$ is the admissible control set.

Although several objective functionals have been proposed (see, for instances, $[1,2,3]$ ) we seek an optimal control relative to the above presented immune-tumor growth models aiming to minimize a combined function of the total number of tumor cells over the entire treatment and a chemotherapeutic drug administration, subject to the state equations (3) ((4) or (5)) and initial conditions (2). Thus, the objective to be minimized is

$$
\min J(u(\cdot)) \equiv \int_{0}^{t_{f}}\left(w_{1} T(t)+\frac{1}{2} w_{2} u(t)^{2}\right) d t,
$$

where $w_{1}$ and $w_{2}$ are positive weights. If $t_{f}$ is fixed and specified, the control problem reflects a treatment process with a fixed duration. The first term represents the tumor cell population over time and the second term reflects the severity of the negative effects of the drug on body. Tumor cell population and control function have different magnitudes, therefore they are balanced by using the weights $w_{1}=5$ and $w_{2}=10$.

\section{NUMERICAL RESULTS}

In order to compare the dynamics of the three tumor growth models via optimal control we use a direct sequential procedure. The optimal control problem is reformulated into the Mayer form and the set of differential equations is integrated by the Matlab function ode15s. The sequentially-discretized problem is solved by the fmincon function from the Matlab Optimization Toolbox ${ }^{\mathrm{TM}}$. The numerical experiments were carried out on a PC Intel Core 2 Duo Processor E7500 with $2.9 \mathrm{GHz}$ and $4 \mathrm{~Gb}$ of memory RAM.

To make the models more realistic, we restrict some parameter values: (i) tumor cells grow faster than normal cells $r_{T}>r_{N}$ and $b_{T} \approx b_{N}$; (ii) the competition effect on immune cells is more effective than on tumor cells $c_{I}<c_{T}$; (iii) $a_{N}<a_{I} \ll a_{T}$ because drug treatment should be more effective in killing tumor cells than in killing the other cells. The time interval $\left[0, t_{f}\right]$ days is discretized using equally spaced subintervals of 0.125 (3 hours). The parameter values used for our experiments, as well as the initial values, are mostly taken from [3]. The other values are assumed for our experiments:

$$
\begin{aligned}
& t_{f}=20 \text { (days) } \\
& b_{T}=5 \times 10^{-9} / \text { cells } \\
& c_{N}=3.422 \times 10^{-10} / \text { cells/day } \\
& a_{N}=1 \times 10^{-5} / \text { day } \\
& \begin{aligned}
& r_{T}= 0.044 / \text { day } \\
& b_{N}= 1 \times 10^{-10} / \text { cells } \\
& c_{I}= 9 \times 10^{-7} / \text { cells/day } \\
& a_{I}=2 \times 10^{-4} / \text { day } \\
& d_{I}=1 \times 10^{2} \mathrm{mg}
\end{aligned} \\
& r_{N}=0.0245 / \text { day } \\
& c_{T}=1.101 \times 10^{-5} / \text { cells } / \text { day } \\
& a_{T}=0.08 / \text { day } \\
& d_{T}=3 \mathrm{mg} \\
& d_{N}=5 \times 10^{6} \mathrm{mg} \\
& d_{D}=0.01 / \text { day } \\
& \rho=6.2 \times 10^{-3} \\
& \alpha=2 \times 10^{-1} \\
& b_{I}=0.0412 / \text { day } \\
& T(0)=5 \text { units of cells } \\
& N(0)=3 \text { units of cells }
\end{aligned}
$$

We present the time series plots of tumor, normal and immune cells on the first row of Figure 1 for the three models. From the figure, we conclude that the tumor cell population level obtained by model (4) is lower than the levels obtained by the other models and is reduced to near zero. The plots on the second row of the figure show the optimal 

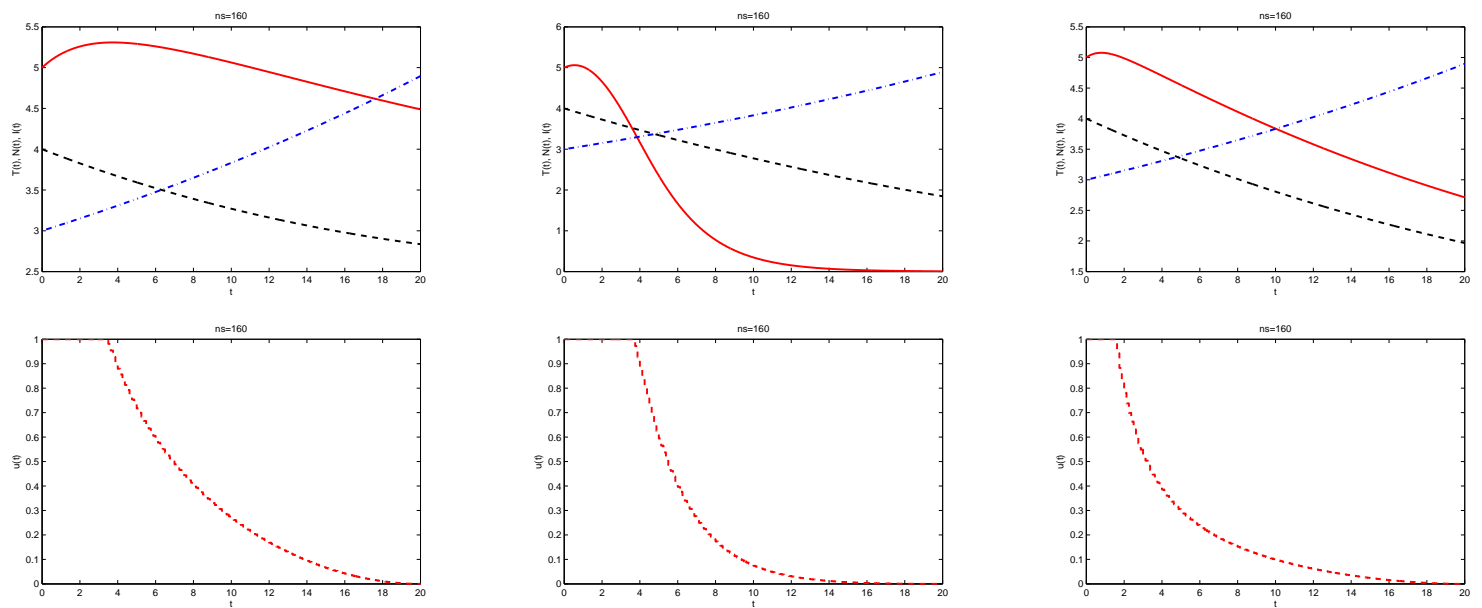

FIGURE 1. Plots of (i) $\mathrm{T}(\mathrm{t})$ (solid lines), $\mathrm{N}(\mathrm{t})$ (dash-dotted lines), $\mathrm{I}(\mathrm{t})$ (dashed lines) - on the top; (ii) $\mathrm{u}(\mathrm{t})$ (dashed lines) - on the bottom. Plots on the left correspond to the model (3), plots in the middle correspond to the model (4) and the plots on the right are from the model (5).

TABLE 1. Final results produced by fmincon, using $I(0)=4$ units of cells.

\begin{tabular}{|c|c|c|c|c|c|c|c|c|c|c|c|}
\hline model & $J\left(u\left(t_{f}\right)\right)$ & Nfeval & time (sec.) & model & $J\left(u\left(t_{f}\right)\right)$ & Nfeval & time (sec.) & model & $J\left(u\left(t_{f}\right)\right)$ & Nfeval & time (sec.) \\
\hline (3) & 403.32 & 2093 & 2231 & (4) & 159.20 & 1932 & 2292 & (5) & 528.90 & 1610 & 1721 \\
\hline
\end{tabular}

control diagram. We may conclude that the drug is more important at the beginning of the treatment than it is at the end. We also include Table 1 to show the final statistics produced by the fmincon solver when computing the optimal control. The model (4) has produced the least objective function value.

\section{ACKNOWLEDGMENTS}

The authors would like to thank the financial support from FCT (Fundação para a Ciência e Tecnologia, Portugal) in the scope of the projects: PEst-OE/MAT/UI0013/2014 and PEst-UID/CEC/00319/2013.

\section{REFERENCES}

1. L.G. De Pillis, A. Radunskaya, Journal of Theoretical Medicine, 3 79-100 (2001).

2. L.G. De Pillis, A. Radunskaya, Mathematical and Computer Modelling, 37 1221-1244 (2003).

3. S. Sharma, G.P. Samanta, Journal of Nonlinear Dynamics, 2013 Article ID 608598, 13 pages (2013).

4. Z. Liu, C. Yang, Computational and Mathematical Methods in Medicine, 2014, Article ID 172923, 12 pages (2014).

5. S.T.R. Pinho, D.S. Rodrigues, P.F.A. Mancera, Canadian Applied Mathematics Quarterly, 19(4) 369-828 (2011). 